\title{
МЕТОД ОЦЕНКИ СТРУКТУРНЫХ СОСТОЯНИЙ В ВОДНЫХ КОЛЛОИДНЫХ СИСТЕМАХ
}

Горленко Николай Петрович д.т.н., профессор Томский государственный архитектурностроительный университет Сидоренко Галина Николаевна к.б.н., доцент Лаптев Борис Иннокентьевич д.б.н., профессор Nove tehnologije d.o.o

Аннотация: Методом диэлектрометрии выявлены структурные особенности в водных растворах хлорида железа, коллоидных частиц гидрозоля железа и воды. Показано, что учет частот собственных гармонических колебаний различных по природе структурных образований позволяет качественно характеризовать особенности их структурных состояний в водных средах.

Ключевые слова: Коллоидные растворы, вода, структура жидких сред, диэлектрометрия, частота собственных колебаний, диполь-дипольные, иондипольные, ион-ионные взаимодействия.

\section{A METHOD FOR ASSESSING STRUCTURAL STATES IN AQUEOUS COLLOIDAL SYSTEMS}

\author{
Gorlenko Nikolay Petrovich \\ Sidorenko Galina Nikolaevna \\ Laptev Boris Innokentievich
}

Abstract: The dielectrometry method revealed structural features in aqueous
solutions of iron chloride, colloidal particles of iron and water hydrosol. It is shown 
that taking into account the frequencies of natural harmonic oscillations of structural formations of different nature makes it possible to qualitatively characterize the features of their structural states in aqueous media.

Key words: Colloidal solutions; water; structure of liquid media; dielectrometry; natural oscillation frequency; dipole-dipole, ion-dipole, ion-ion interactions.

При исследовании свойств воды прослеживается «явная» способность молекул воды существовать в виде отдельных диполей и «скрытая» в виде их способности объединяться в одно кинетическое целое. «Явная» способность отражается в прямых экспериментальных данных, описывающих различные физические свойства отдельных молекулы воды (диполей), таких, например, как заряд, размер и др., а «скрытая» заключается в многочисленных косвенных доказательствах проявления ассоциативных (кооперативных) свойств молекул воды, что в настоящее время является предметом дискуссий [1-5].

На наш взгляд, возможность существования ассоциатов оправдывается проявлением энергии водородных и дипольных связей между молекулами воды. Несмотря на то, что средняя энергия ион-дипольных и диполь-дипольных связей в воде меньше по сравнению с водородной связью, однако они имеют один и тот же порядок, что является достаточным условием для конкуренции с тепловой энергией броуновского движения.

Основой теоретического обоснования полученных ниже экспериментальных данных является предположение, что молекулы воды могут существовать как в виде отдельных диполей, так и в виде их объединенных образований (кластеры, ассоциаты и др.), а во время взаимодействия друг с другом они ведут себя как гармонические осцилляторы. При этом, как было показано ранее [6], объединение молекул в количестве $10^{3}$ и более единиц и более при комнатной температуре и нормальном давлении, является достаточным условием их стабильного существования в водной среде.

Учитывая это, а также в силу различия линейных и объемных размеров структурных единиц, каждая структура должна иметь собственную частоту колебаний. Так как коллоидные структуры имеют вполне определенную структуру, отличающуюся от структурной организации воды или водных растворов, то представляет интерес провести сравнительную оценку структурных особенностей таких систем. При этом, одним из информативных 


\section{ИННОВАЦИОННОЕ РАЗВИТИЕ СОВРЕМЕННОЙ НАУКИ:

методов оценки структурных изменений в воде и водных растворах является метод диэлектрометрии.

В экспериментах для измерения электрической емкости воды, растворов $\mathrm{FeCl}_{3}$ и их золей использована измерительная ячейка, которая включает стандартную стеклянную пробирку диаметром 20 мм и длиной 200 мм, в которую помещают исследуемые жидкости, а также две обкладки конденсатора из немагнитного материала (площадью $22 \mathrm{~cm}^{2}$ каждая), находящиеся на пробирке (рис. 1). Обкладки конденсатора смещены относительно друг друга в параллельных плоскостях и не имеют поверхности, расположенной напротив друг друга.

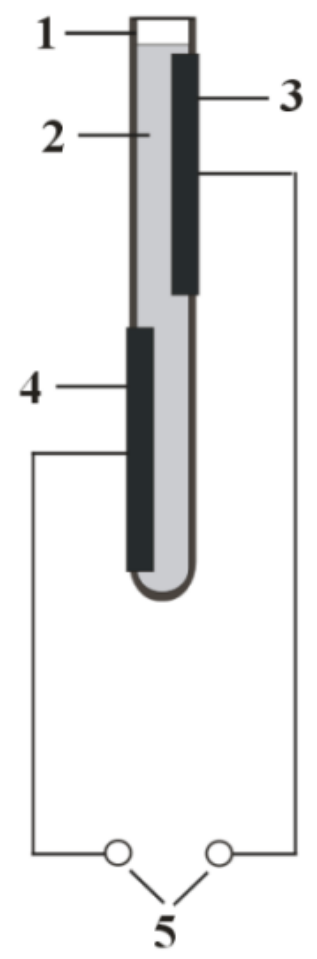

Рис. 1. Измерительная ячейка для измерения электрической емкости воды, растворов $\mathrm{FeCl}_{3}$ и их золей. 1 - емкость для жидкости, 2 - исследуемая жидкость, 3 и 4 - обкладки конденсатора на измерительной ячейке, которые смещены относительно друг друга в параллельных плоскостях и не имеют поверхности, расположенной напротив друг друга; 5 - клеммы для подключения сигнала от генератора синусоидальных колебаний 


\section{ИННОВАЦИОННОЕ РАЗВИТИЕ СОВРЕМЕННОЙ НАУКИ: ТЕОРИЯ, МЕТОДОЛОГИЯ, ПРАКТИКА}

Напряжение к измерительным ячейкам подается от генератора синусоидальных колебаний AFG 2025, сигнал с ячеек усиливается инструментальным усилителем на основе микросхемы AD8067 и измеряется на осциллографе PDS5022S. Величина напряжения генератора синусоидальных колебаний, подводимого к измерительной ячейке, уменьшается обратно пропорциональна его частоте. Это, во-первых, уменьшает зависимость плотности тока через измерительную ячейку от его частоты, что снижает влияние величины реактивного тока на структуру жидкостей. Во-вторых, такая конструкция измерительной ячейки увеличивают динамический диапазон изменения сигнала при изменении структуры водосодержащих сред. При этом плотность тока на обкладках конденсатора при измерении емкости не превышает $50 \mathrm{HA} / \mathrm{cm}^{2}$, что обеспечивает минимальное внешнее воздействие на жидкость, а частота изменяется в пределах от 1 кГц до 3000 кГц. Детали методики описаны ранее в работе [7].

В опытах использована дистиллированная вода с проводимостью при $20 \mathrm{C}$ от 1,2 до 2 мкСм/см, а также водные растворы хлорида железа в концентрациях $1 \times 10^{-3} \mathrm{M}$ и $1 \times 10^{-2} \mathrm{M}$. Золь гидроксида железа получали путем кипячения раствора $\mathrm{FeCl}_{3}$ указанных выше концентраций в течение 3 минут до образования жидкости красно-коричневого цвета. Исследования проведены при температуре жидкостей $20^{\circ} \mathrm{C}$.

На рисунке 2 приведены результаты изменения электрической емкости в зависимости от частоты реактивного тока в золях гидроксида железа, полученных после нагревания и последующего остывания раствора $\mathrm{FeCl}_{3}$ до температуры $20{ }^{\circ} \mathrm{C}$.

Оказалось, что электрическая емкость золя гидроксида железа, по сравнению с соответствующим водным раствором $\mathrm{FeCl}_{3}$ в концентрации $1 \times 10^{-3}$ М возрастала на частотах от 300 кГц до 3000 кГц, (Р<0,001 во всех случаях). Аналогичные изменения параметров наблюдались и при исследовании золя гидроксида железа, по сравнению с соответствующим водным раствором $\mathrm{FeCl}_{3}$ в концентрации $1 \times 10^{-2} \mathrm{M}$. В этом случае электрическая емкость золя возрастала на частотах 1000 кГц и 3000 кГц, $(\mathrm{P}<0,001$ во всех случаях $)$. 


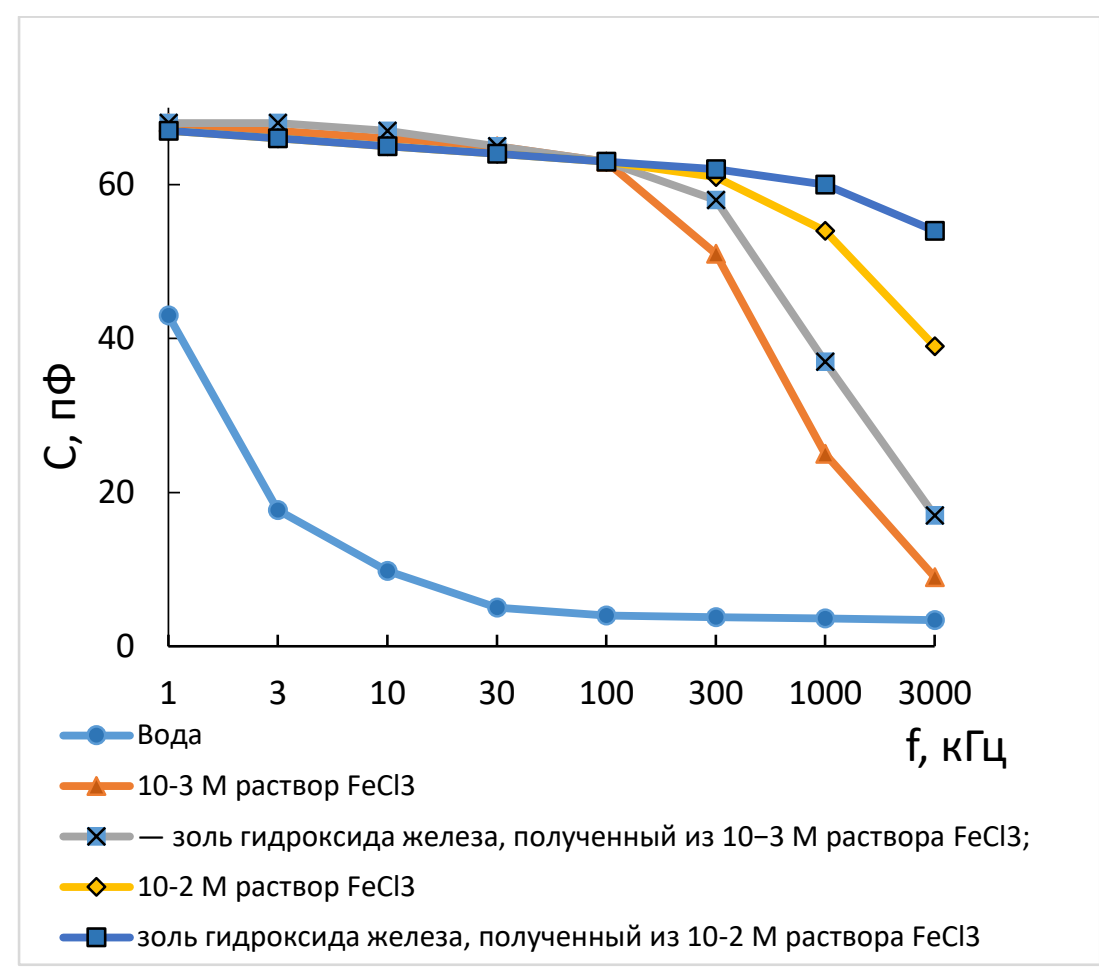

Рис. 2. Зависимость электрической емкости дистиллированной воды, водных растворов $\mathrm{FeCl}_{3}$ и золей гидроксида железа, полученных из $10^{-3} \mathrm{M}$ и из $10^{-2} \mathrm{M}$ растворов $\mathrm{FeCl}_{3}$ от частоты реактивного тока

Очевидно, что значения электрической емкости исследуемых систем коррелируют с частотой собственных колебаний структурных образований в воде и водных растворах. Если линейные или объемные структуры успевают синхронно ориентироваться относительно изменения частоты электрического поля, то значение емкости $(C)$ увеличивается; если наблюдается запаздывание, то значение $C$ уменьшается; когда структуры не реагируют на частоту электрического поля, то значение емкости остается постоянным.

В качестве основных структур, присутствующих в исследуемых системах, следует выделить следующие:

1. Структура золя гидроксида железа (рис. 3).

$\left\{\mathrm{m}\left[\mathrm{Fe}(\mathrm{OH})_{3}\right]_{\mathrm{n}} \mathrm{Fe}^{3+}(3 \mathrm{n}-\mathrm{x}) \mathrm{Cl}^{-} 3 \mathrm{Cl}^{-}\right\}^{3 \mathrm{x}+} \times 3 \mathrm{Cl}^{-}$ 


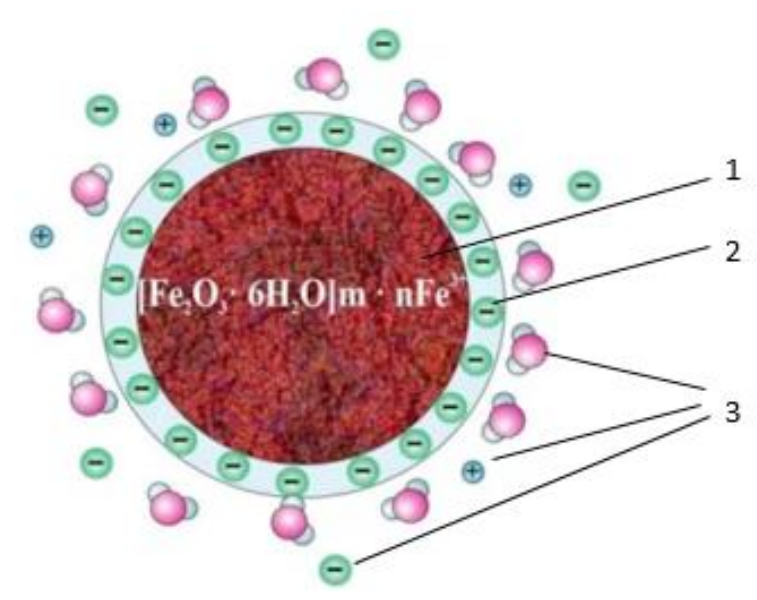

Рис. 3. Мицелла гидроксида железа, полученного методом гидролиза. 1- ядро, 2- адсорбционный слой, образованный ионами $\mathrm{Cl}^{-}, 3$ - диффузный слой, образованный ионами хлора, молекулами воды и противоионами

2. Структура гидратированных ионов железа и хлора (рис. 4):
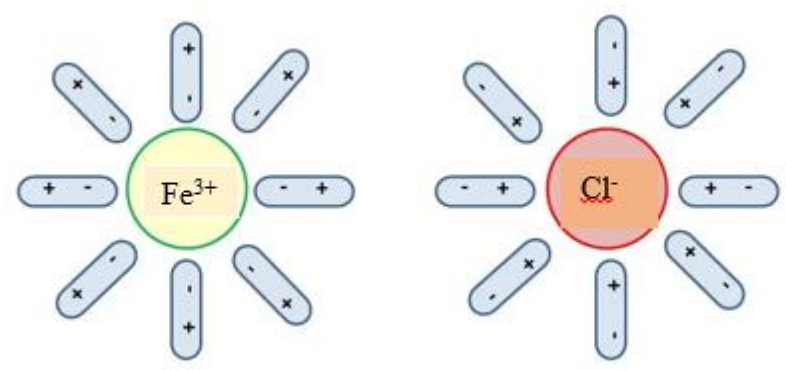

Рис. 4. Структура гидратированных ионов железа и хлора

3. Структурные образования молекул воды (рис.5).

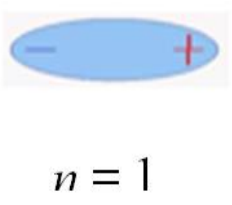

1
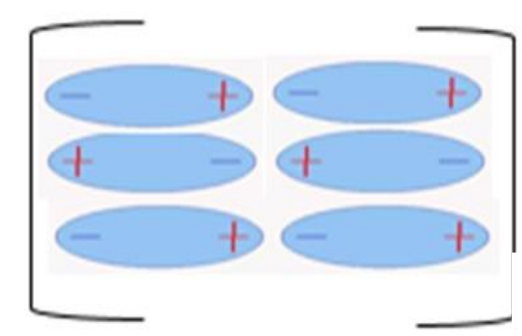

2

Рис. 5. Структуры молекул воды: 1 - отдельные диполи;

2 - диполи воды, организованные в ассоциат в количестве $\boldsymbol{n}$ единиц. 


\section{ИННОВАЦИОННОЕ РАЗВИТИЕ СОВРЕМЕННОЙ НАУКИ:

Известно, что частота собственных гармонических колебаний различных материальных образований при достаточно малых отклонениях от положения равновесия зависит от их массы, температуры окружающей среды и может быть рассчитана с помощью приведенных ниже уравнения (1) [8]:

$$
f=\frac{1}{2 \pi} \sqrt{K / m},
$$

где $f$ - частота собственных колебаний тела, $K=F / l$ - коэффициент пропорциональности, представляющий собой упругую силу, приходящуюся на единицу длины связи, $m$ - масса колеблющегося ассоциата, проявляющего кооперативные свойства.

В таблице приведены габаритные размеры и массы структурных составляющих исследуемых растворов.

Таблица 1

Диаметры и массы структурных составляющих растворов

\begin{tabular}{|c|c|c|c|c|c|c|c|}
\hline \multicolumn{8}{|c|}{ Размеры и массы структурных составляющих растворов } \\
\hline \multicolumn{2}{|c|}{ Молекула воды } & \multicolumn{2}{|c|}{ Ион железа $\mathrm{Fe}^{3+}$} & \multicolumn{2}{|c|}{ Ион хлора, $\mathrm{Cl}^{-}$} & \multicolumn{2}{|c|}{ Мицелла гидроксида } \\
\hline диаметр, & масса, & диаметр, & масса, & диаметр, & масса, & диаметр, & \multirow{2}{*}{ масса, кг } \\
м & кг & м & кг & м & кг & м & \\
\hline $2.8 \times 10^{-10}$ & $3 \times 10^{-23}$ & $2.6 \times 10^{-10}$ & $9.3 \times 10^{-23}$ & $2.0 \times 10^{-10}$ & $5.8 \times 10^{-23}$ & $10^{-7}-10^{-9}$ & $9.4 \times 10^{-20}$ \\
\hline
\end{tabular}

Учитывая, что между молекулами воды осуществляется дипольдипольное взаимодействие, между ионами железа, хлора и их гидратными оболочками ион-дипольные взаимодействия, а между мицеллами гидроксида железа ион-ионное взаимодействие рассчитаем частоты собственных колебаний структурных образований по уравнению (1), используя данные таблицы 1.

Сила диполь-дипольного взаимодействия определяется зависимостью [9].

$$
F_{\partial-\partial}=\frac{n \mu n \mu}{4 \pi \varepsilon_{0} \varepsilon_{1} r^{4}}=\frac{n^{2} \mu^{2}}{4 \pi \varepsilon_{0} \varepsilon_{1} r^{4}},
$$

где $\mu$ - дипольный момент молекулы воды $\left(6,1 * 10^{-30}\right.$ Кл*м), $\varepsilon_{0}-$ электрическая постоянная $\left(8,85 * 10^{-12} \Phi / \mathrm{M}\right), \varepsilon_{l}$ - относительная диэлектрическая проницаемость воды (для связанных молекул в единое кинетическое образование принимается равным 1), $r$ - радиус взаимодействующих тел, $n-$ число контактов на единицу поверхности. 


\section{ИННОВАЦИОННОЕ РАЗВИТИЕ СОВРЕМЕННОЙ НАУКИ:

При $\mathrm{n}=1$ для двух взаимодействующих диполей:

$F_{\partial-\partial}=\left(6,1 * 10^{-30}\right)^{2} / 4 \times 3,14 \times 8,85 * 10^{-12} \times 1 \times\left(1,4 * 10^{-10}\right)^{4}=8,8 * 10^{-10} \mathrm{H}$.

При $\mathrm{n}=10^{3}, F_{\partial-\partial}=8,8 \times 10^{-4} \mathrm{H}$. При $\mathrm{n}=10^{6}, F_{\partial-\partial}=8,8 \times 10^{2} \mathrm{H}$. При $\mathrm{n}=10^{9}$, $F_{\partial-\partial}=8,8 \times 10^{8} \mathrm{H}$.

Сила ион - дипольного взаимодействия определяется по формуле:

$$
F_{u-d}=\frac{n q n \mu}{4 \pi \varepsilon_{0} \varepsilon r^{3}},
$$

где $q$ - заряд электрона $1,6 \times 10^{-19}$ Кл, $r=1,5 \times 10^{-5} \mathrm{M}, \varepsilon=1$ (при контактном взаимодействии диполя воды с ионом [9].

Для $n=1$ при взаимодействии иона железа и диполя воды (проявляется ион-дипольное взаимодействие):

$$
F_{u-\delta}=\left(1,6 * 10^{-19} \times 6,1 * 10^{-30}\right) / 4 \times 3,14 \times 8,85 * 10^{-12} \times 1 \times\left(1,3 * 10^{-10}\right)^{3}=4,2 * 10^{-9} \mathrm{H} .
$$

Для $n=1$ при взаимодействии иона хлора и диполя воды сила иондипольного взаимодействия имеет приблизительно такой же порядок, как и при взаимодействии иона железа с молекулой воды.

Сила ион-ионного взаимодействия определяется выражением [9]:

$$
F_{u-\partial}=\frac{n q n q}{4 \pi \varepsilon_{0} \varepsilon r^{2}}
$$

Логично предположить, что ион-ионное взаимодействие для коллоидных частиц железа может осуществляться через взаимодействие электрокинетических потенциалов, возникающих на границе адсорбционного слоя (рис.3).

При $\mathrm{n}=1$ для двух взаимодействующих мицелл гидроксида железа:

$F_{u-u}=\left(1,6 * 10^{-19}\right)^{2} / 4 \times 3,14 \times 8,85 * 10^{-12} \times 1 *\left(10^{-9}\right)^{2}=2,4 * 10^{-6} \mathrm{H}$.

Так как мицеллы гидроксида железа проявляют силы отталкивания, условно примем, что рассчитанное значение $F_{u-u}$ соответствует минимальной силе отталкивания, обеспечивающей стабильное состояние коллоидного раствора.

С учетом значений сил взаимодействия для каждого из структурных элементов водного раствора рассчитаем коэффициент пропорциональности (К), представляющий собой упругую силу, приходящуюся на единицу длины связи.

$$
\begin{aligned}
& \mathrm{K}_{\text {д-д }}=8,8 * 10^{-10} / 2,8 * 10^{-10}=3,1 \mathrm{H} / \mathrm{M} ; \quad \mathrm{K}_{\text {и-д }}=4,2 * 10^{-9} / 2,6 * 10^{-10}=16 \mathrm{H} / \mathrm{M} ; \\
& \mathrm{K}_{\text {и-и }}=2,4 * 10^{-6} / 1,0 \times 10^{-7}=2,4 \mathrm{H} / \mathrm{M} .
\end{aligned}
$$




\section{ИННОВАЦИОННОЕ РАЗВИТИЕ СОВРЕМЕННОЙ НАУКИ:

Отсюда, в соответствии с уравнением (1), масса структурных единиц в водном растворе в зависимости от частоты их собственных колебаний должна соответствовать выражению:

$$
m=\frac{K}{4 \pi f^{2}}
$$

Рассчитаем массы структурных образований, которые способны успевать ориентироваться синхронно с изменением частоты электрического поля согласно значениям рис. 2.

1. Для молекул воды (проявляется диполь-дипольное взаимодействие). Изменение частоты наблюдается в пределах 1- 30 кГц). Тогда масса взаимодействующих диполей будет равна:

$$
m=\frac{K}{4 \pi f^{2}}=\frac{3,1}{4 \times 3,14 \times\left(1 * 10^{3}-3 * 10^{4}\right)^{2}}=2 * 10^{-5}-1,8 * 10^{-7} \text { кг. }
$$

Так как масса одной молекулы воды составляет $3,0 * 10^{-26}$ кг, то с учетом $30 \%$ пустот в структурной организации воды, количество диполей воды, связанных в ассоциат, должно составлять $0,3 \times 3,0 * 10^{-26} / 1,8 * 10^{-7}=5,0 * 10^{21}$ частиц. Эти расчеты, с одной стороны, позволяют утверждать о справедливости континуальной модели структуры воды, согласно которой в воде практически отсутствуют молекулы с разорванными водородными связями, т.е. все молекулы воды структурно и энергетически эквивалентны. Но с другой стороны, сохраняется и высокая доля с другим количеством объединенных в ассоциат молекул. В табл. 2 приведены оценочные расчеты частот собственных колебаний молекул с различным количеством взаимодействующих диполей воды [6].

Таблица 2

Собственные частоты колебаний одинаковых по размерам ассоциатов при их взаимодействии на расстояниях, равных диаметру

\begin{tabular}{|c|c|c|c|c|c|c|c|}
\hline $\begin{array}{l}\text { Количество } \\
\text { молекул воды } \\
\text { в ассоциате }\end{array}$ & $\begin{array}{l}r, \\
\text { м }\end{array}$ & $\begin{array}{c}n, \\
\text { отн. ед. }\end{array}$ & $\begin{array}{l}m, \\
\text { кг }\end{array}$ & $\begin{array}{c}F_{\partial-\partial,} \\
\mathrm{H}\end{array}$ & $\begin{array}{l}l, \\
\mathrm{~m}\end{array}$ & $\begin{array}{c}k, \\
\kappa \Gamma / \mathrm{c}^{2}\end{array}$ & $\begin{array}{l}f, \\
\Gamma_{ц}\end{array}$ \\
\hline $10^{0}$ & $1,4 \times 10^{-10}$ & $1,0 \times 10^{0}$ & $3,0 \times 10^{-26}$ & $8,3 \times 10^{-10}$ & $2,8 \times 10^{-10}$ & 2,96 & $1,6 \times 10^{14}$ \\
\hline $10^{3}$ & $6,2 \times 10^{-9}$ & $2,0 \times 10^{3}$ & $9,9 \times 10^{-22}$ & $2,8 \times 10^{-10}$ & $1,2 \times 10^{-8}$ & 2,33 & $7,7 \times 10^{10}$ \\
\hline $10^{6}$ & $2,2 \times 10^{-8}$ & $2,5 \times 10^{4}$ & $4,4 \times 10^{-20}$ & $8,5 \times 10^{-9}$ & $4,4 \times 10^{-8}$ & 0,19 & $3,3 \times 10^{8}$ \\
\hline $10^{9}$ & $1,3 \times 10^{-7}$ & $8,6 \times 10^{5}$ & $9,1 \times 10^{-18}$ & $5,1 \times 10^{-11}$ & $2,6 \times 10^{-7}$ & $2,0 \times 10^{-4}$ & $5,3 \times 10^{6}$ \\
\hline $10^{12}$ & $1,1 \times 10^{-6}$ & $6,1 \times 10^{7}$ & $5,6 \times 10^{-15}$ & $5,0 \times 10^{-13}$ & $2,2 \times 10^{-6}$ & $2,3 \times 10^{-7}$ & $1,0 \times 10^{3}$ \\
\hline
\end{tabular}
кинетических образований 


\section{ИННОВАЦИОННОЕ РАЗВИТИЕ СОВРЕМЕННОЙ НАУКИ: ТЕОРИЯ, МЕТОДОЛОГИЯ, ПРАКТИКА}

Как видно из табл. 2, отдельные диполи воды должны синхронно ориентироваться относительно силовых линий электрического поля во всем диапазоне исследуемых частот, а ассоциаты из $10^{12}$ единиц и выше не будут успевать реагировать на изменение частоты (для этих случаев значение емкости будет оставаться постоянной величиной). Ассоциаты с объединением молекул в количестве от $10^{3}$ до $10^{12}$ единиц будут реагировать на изменение частоты (значение емкости будет снижаться), что и наблюдается экспериментально. Ход кривой для чистой воды (рис. 1) позволяет утверждать, что в воде присутствуют ассоциаты различных размеров (от отдельных диполей до структурных образований в количестве $10^{12}$ единиц)

2. Для гидратированных ионов (проявляется ион-дипольное взаимодействие между ионами железа или хлора и диполями воды).

$$
m=\frac{K}{4 \pi f^{2}}=\frac{16}{4 \times 3,14 \times\left(1 * 10^{5}-3 * 10^{6}\right)^{2}}=1,3 * 10^{-10}-4 * 10^{-11} \text { кг. }
$$

Ионы железа или хлора образуют первичную и вторичную гидратные оболочки. При этом количество молекул воды в первичной оболочке составляет порядка 6-10 единиц, а во вторичной может превышать несколько сотен. Кроме указанных структурных образований в водном растворе хлорида железа образуются ассоциаты из противоположно заряженных гидратированных ионов по типу ион-ионного взаимодействия. На способность образовывать в водном растворе такого типа структурные образования косвенно указывает различный ход кривых для растворов хлорида железа различной концентрации (рис. 1). Так как масса одной молекулы хлорида железа (III) составляет $3,3 * 10^{-24}$ кг, то количество молекул в одном ассоциате должно составлять примерно $10^{13}-10^{14}$ единиц, что, по нашему мнению, является результатом как процессов гидратации ионов, так и их корреляционного взаимодействия с образованием многомерной структуры.

3. Для мицелл коллоидного раствора.

$$
m=\frac{K}{4 \pi f^{2}}=\frac{2,4}{4 \times 3,14 \times\left(3 \times 10^{6}\right)^{2}}=2,0 \times 10^{-13} \text { кг. }
$$

Так как средняя молярная масса мицеллы гидрозоля гидроксида железа составляет 56500 г/моль, то только атомов железа в мицелле не менее 530 единиц. С учетом адсорбционного и диффузионных слоев коллоидной частицы (рис. 3), это количество возрастает до нескольких сотен тысяч молекул. 


\section{ИННОВАЦИОННОЕ РАЗВИТИЕ СОВРЕМЕННОЙ НАУКИ:

Если принять, что средний размер коллоидной частицы железа $10^{-8} \mathrm{M}$, a eе форма представляет собой шар, то ее объем равен $3,1 * 10^{-16} \mathrm{~m}^{3}$. Принимая плотность мицеллы равной 1000 кг/м³ , получаем массу частиц в таком структурном образовании, равную порядка $10^{-13}$ кг.

Сравнивая экспериментально полученные данные и теоретические расчеты по массам колеблющихся коллоидных частиц, следует отметить их близкую сопоставимость.

\section{Выводы}

1. Описанный в работе методический подход с использованием метода диэлектрометрии может в дальнейшем использоваться как метод оценки структурных состояний в водных коллоидных системах.

2. Метод диэлектрометрии позволяет выявить изменения, происходящие в растворах солей хлорида железа (III) при их переходе в золь гидроксида железа.

\section{Список литературы}

1. Ho M-W. Large Supramolecular Water Clusters Caught on Camera - A Review // Water. - 2013. - T.6. - P. 1-12.

2. Сыроешкин А.В., Смирнов А.Н., Гончарук В.В. и др. Вода как гетерогенная структура // Электронный журнал «Исследовано в России». 2006. - C. 843-854. http://zhurnal.ape.relarn.ru/articles/2006/088.pdf.

3. Sidorenko G., Brilly M., Laptev B., Gorlenko N., Antoshkin L., Vidmar A., Kryžanowski A.. The Role of Modification of the Structure of Water and WaterContaining Systems in Chang-ing Their Biological, Therapeutic, and Other Properties. // Water. - 2021. - V.13. - P. 2441. doi.org/10.3390/w13172441/

4. Сидоренко Г.Н. Лаптев Б.И. Горленко Н.П., Антошкин Л.В. Оценка изменений структурной организации воды и водосодержащих систем при различных воздействиях с использованием электрофизических методов и термометрии. / Материалы Межвузовского международного конгресса. Москва: Издательство Инфинити. - 2021. - C. 96-109. DOI 10.34660/INF.2021.21.71.015

5. Фаращук Н.Ф., Рахманин Ю.А. Вода - структурная основа адаптации. Москва: Смоленск. - 2004. - 151 с. 


\section{ИННОВАЦИОННОЕ РАЗВИТИЕ СОВРЕМЕННОЙ НАУКИ:

6. Лаптев Б.И., Сидоренко Г.Н., Горленко Н.П., Саркисов Ю.С., Антошкин Л.В. Процессы структурообразования в воде и в водных растворах // Вода и экология. Проблемы и решения. - 2012. - № 2/3. - С. 26 -34.

7. Сидоренко Г. Н., Лаптев Б. И., Горленко Н. П., Саркисов Ю. С., Антошкин Л. В. Динамика структурной организации воды и водных растворов в диапазоне частот от 100 Гц до 3 мГц // Водоочистка. Водоподготовка. Водоснабжение. - 2016. - № 9 (105). - С. 40-45. 15.

8. Бабаков И.М. Теория колебаний. Москва: Наука. - 1968. - 560 с.

9. Эрдей - Груз Т. Явления переноса в водных растворах. Москва: Наука. - 1976. -591 c. 